\title{
Teorias del crecimiento endogeno: lectura desde los territorios de la periferia
}

\author{
CARLOS A. DE MATTOS
}

"La economía en la que vivimos está más próxima a la visión de Kaldor, la de un mundo dinámico guiado por procesos acumulativos, que la del modelo típico de rendimientos constantes a escala”

Krugman (1992:15)

\section{Desigualdades interregionales, ¿convergencia o divergencia?}

F

UE EN EL PERÍODO que siguió a la Segunda Guerra Mundial cuando la existencia de desigualdades económicas y de bienestar entre diferentes regiones comenzó a ser considerada como un problema que debía ser objeto de análisis y de corrección. Desde entonces, numerosos estudios, tanto de carácter teórico cuanto empírico, han originado una prolongada controversia, aún inconclusa, acerca de si con un mayor crecimiento dichas desigualdades tenderían hacia la convergencia o hacia la divergencia.

El análisis de las argumentaciones en pro de una u otra postura muestra que ellas buscaron respaldo en las hipótesis, supuestos y explicaciones suministradas por diferentes modelos teóricos sobre los orígenes y la dinámica del crecimiento económico.

Por otra parte, aún cuando buena parte de estos modelos se desenvuelven en el campo de la teoría positiva, cuyo alcance es esencialmente descriptivo, explicativo y predictivo, sus principales conclusiones habitualmente han terminado siendo adoptadas como base para formular políticas con el propósito de atenuar esas desigualdades y/o fortalecer el camino hacia la convergencia. Así lo indica nítidamente la experiencia de gestión pública observable en este período, donde los aportes de los modelos de crecimiento disponibles suministraron el indispensable fundamento para las políticas respectivas. 
Lo que no significa que dichas políticas siempre hayan guardado estricta correspondencia con los fundamentos teóricos escogidos, puesto que en buena parte de los casos solamente contemplaron de manera general sus lineamientos básicos, adaptados a los condicionamientos impuestos por las especificidades de cada situación concreta. Como señala Cuadrado Roura (1995: 6), “la 'praxis' política, entonces, después y ahora, no siempre arranca ni está en armonía con un 'modelo' o esquema teórico bien definido, aunque algunos elementos de las teorías disponibles justifican (o sirven para justificar o apoyar) las medidas adoptadas". En definitiva, resulta difícil encontrar ejemplos de estrategias aplicadas para la corrección de las desigualdades o para la promoción del crecimiento que directa o indirectamente no hayan utilizado como soporte las relaciones causales de un marco teórico específico.

Es así, por lo tanto, que desde el momento en que este tema adquirió relevancia, los fundamentos tanto de los discursos favorables a la convergencia o a la divergencia como de las prescripciones normativas para atenuar las disparidades interregionales, han sido extraidos-explícita o implicitamentede los modelos teóricos de crecimiento económico que han estado en boga a lo largo de este periodo. La vigencia de esta tesis aconseja plantear el análisis de los problemas del crecimiento regional (nacional o subnacional) y de la convergencia/divergencia a la luz de la evolución de los modelos formalizados de crecimiento económico (1).

A este respecto se pueden delimitar tres momentos (expuestos en el cuadro) principales en función de la influencia efectiva de las corrientes básicas de pensamiento dominantes en cada uno de ellos (2): el primero, cuando la influencia procedió principalmente de las ideas y recetas keynesianas; el segundo, cuando estas fueron desplazadas por modelos de corte neoclásico y el tercero, actualmente en pleno desarrollo, donde el influjo procede de un conjunto de nuevos modelos, también de origen neoclásico, que privilegian el carácter endógeno del crecimiento (3). La revisión de los hitos centrales de esta historia suministra elementos de juicio importantes para la comprensión de los alcances de la evolución vivida en el período de referencia y para el análisis de sus implicancias.

En esta dirección, en este trabajo se propone explorar los cambios producidos por la aparición de las nuevas teorías neoclásicas en cuanto a las perspectivas de crecimiento para el caso de los territorios de menor desarrollo relativo y sobre las consecuentes previsiones en términos de convergencia o divergencia interregional. A estos efectos, ante todo se analiza la influencia que tuvieron los modelos de crecimiento derivados de las teorías keynesiana y neoclásica en las políticas regionales en el período de la segun- 
da posguerra, como base para caracterizar los cambios que han comenzado a observarse a raíz de la creciente difusión de los modelos de crecimiento endógeno. Con este propósito se caracterizan los principales aportes de estos nuevos modelos y se analizan sus principales implicancias para la definición de políticas regionales de alcance nacional y/o subnacional. Finalmente, se esbozan algunos elementos de juicio para la discusión sobre las perspectivas de crecimiento económico para el caso de territorios de menor desarrollo relativo a la luz de los factores y las explicaciones aportados por las nuevas teorías del crecimiento y se analizan las previsiones sobre convergencia o divergencia en el nuevo escenario emergente.

\section{Desequilibrios}

\section{y divergencia en la dinamica keynesiana}

Cuando el ascendiente del pensamiento keynesiano llegó a su apogeo, sus principales aportes teóricos se manifestaron en un importante desarrollo de nuevas ideas y explicaciones sobre las condiciones y los factores que impulsaban o limitaban el crecimiento económico. Fue así que en los años que siguieron a la Segunda Guerra Mundial hicieron su irrupción diversos modelos concebidos bajo la influencia keynesiana, donde se destacan, entre muchos otros, los formulados por Harrod, Domar, Kaldor, Robinson y Pasinetti. Essos modelos, concentraron básicamente su atención en el papel de la inversión y de los factores que podrían afectarla en una situación donde la evolución de la demanda agregada se ubica como el impulsor fundamental. Más allá de la diversidad de enfoques que es posible encontrar entre los diversos modelos keynesianos y poskeynesianos, una convicción medular subyace en todos ellos: en situación de plena concurrencia, el funcionamiento de las economias de mercado genera desequilibrios y desemboca casi inevitablemente en el desempleo.

$\mathrm{Al}$ poner en cuestión el carácter automático de los ajustes y de la reabsorción de los desequilibrios se infería, como conclusión lógica, la necesidad de una intervención exógena al mercado con el propósito de sostener la demanda y promover el crecimiento y el empleo. Ya Keynes había destacado que el objetivo fundamental de la política económica era el de aminorar el aumento del desempleo, problema que se constituyó en su principal preocupación, así como también en el de buena parte de los economistas que habían vivido los efectos de la Gran Depresión de los años 30. En otras palabras, si los empresarios privados no ahorraban y no realizaban las inversiones necesarias para asegurar los niveles adecuados de empleo, esta tarea debía ser asumida por el sector público. 
Momentos de las teorías del crecimiento

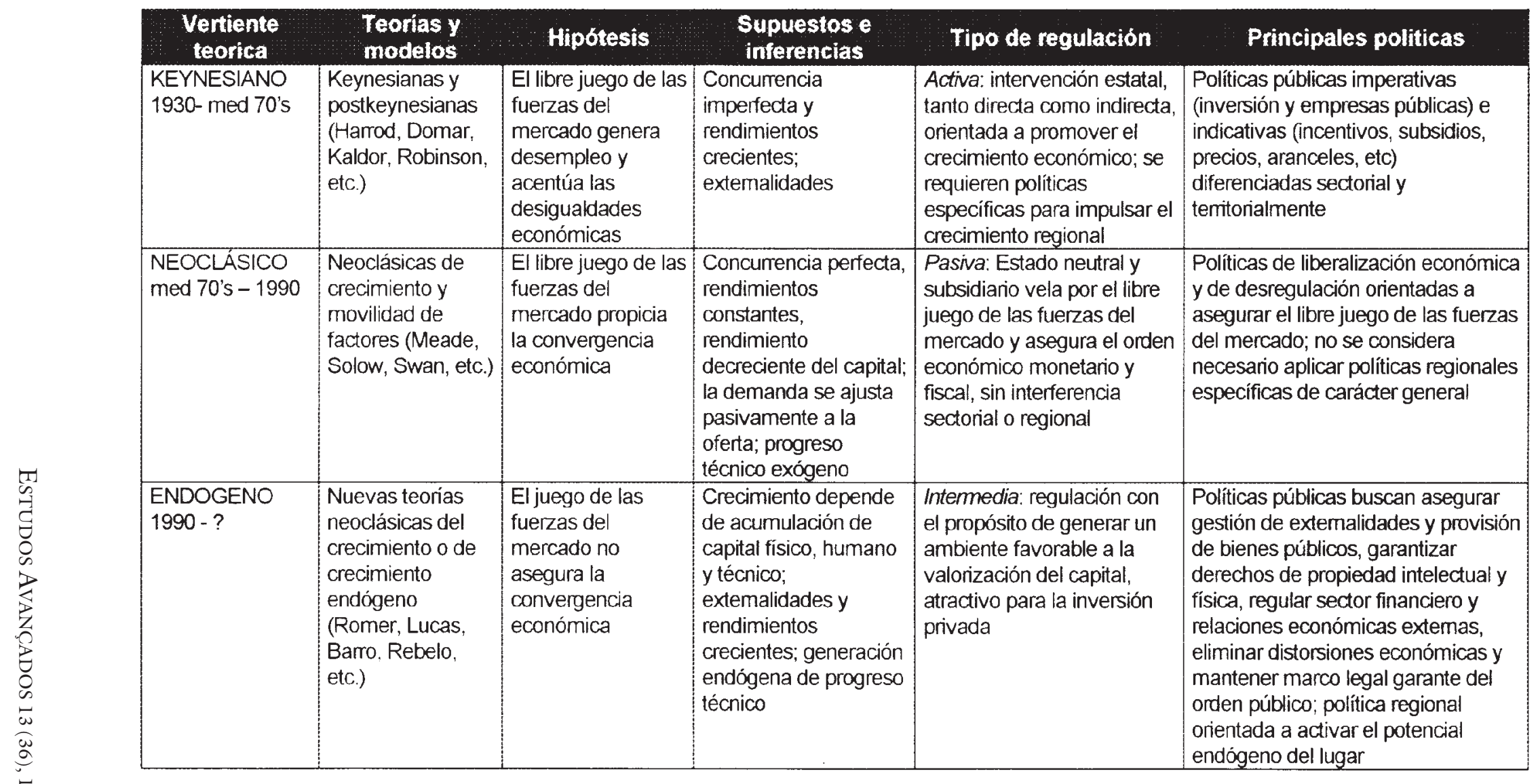


A partir de allí, algunos importantes trabajos realizados en los años de la posguerra por autores directa o indirectamente vinculados a esta corriente, entre los que pueden destacarse Perroux (1955), Myrdal (1957), Hirschman (1958) y Kaldor (1970), propusieron que en ausencia de mecanismos correctores, el libre juego de las fuerzas del mercado llevaba inexorablemente a una intensificación de las desigualdades interregionales. Una conclusión representativa de esta línea de pensamiento fue expresada tajantemente por Myrdal en los términos siguientes: "la idea principal que quiero transmitir es que normalmente el juego de las fuerzas del mercado tiende a aumentar, más bien que a disminuir, las desigualdades entre las regiones" (Myrdal, 1957: 38).

En lo esencial, se suponía que en una economía de libre mercado el crecimiento tendía a beneficiar acumulativamente a los territorios de mayor desarrollo (o centrales) y a perjudicar a los menos desarrollados (o periféricos). A la luz de estos planteamientos - y también de los de la corriente marxista que tuvo una importante influencia en este período - irrumpieron distintas propuestas (causación circular acumulativa, centro-periferia, polos de crecimiento, colonialismo interno), en las que la divergencia aparecía como congénita a la transmisión interregional del crecimiento en una situación regida por el libre juego de las fuerzas del mercado.

Dentro de los cauces de la vertiente keynesiana hacia fines de este período se abrieron paso las teorías del desarrollo económico y social, las que sin duda tomaron sus hipótesis fundamentales de los modelos de crecimiento, si bien los límites entre unos y otras no pueden ser establecidos con precisión (Assidon, 1992: 39). En este nuevo ámbito, economistas como Myrdal, Nurkse, Rosenstein-Rodan y Hirschman, entre otros, procuraron encontrar respuestas a la preocupación que suscitaba la tenaz persistencia del crecimiento divergente.

Bajo el influjo de todo este conjunto de teorías y modelos, se desarrolló lo que se ha denominado como la edad de oro del intervencionismo, en la que la revolución keynesiana aparece como el origen de la convicción de que al fin era posible dominar la actividad económica (Beaud \& Dorstaler, 1993: 93). Fue el momento en que alcanzó sus mejores momentos la gran fe en la razón iluminista, que se manifestó en una confianza plena en la posibilidad de la ingeniería social: se suponía posible avanzar hacia una mayor racionalidad sustantiva (el desarrollo), mediante el ejercicio de una racionalidad formal (la planificación). Así, en esos años, se impuso en diversos lugares del mundo (4), la convicción de que era posible planificar el desarrollo bajo la conducción del Estado, lo que a la larga redundó en una plétora de experiencias, tan diversas como infructuosas. 
En ese ámbito también surgieron los intentos de planificar el desarrollo regional para lo cual, al influjo de las teorías keynesianas del crecimiento, se esbozaron diversas estrategias en las que se entrelazaban ideas como la de la industrialización sustitutiva y la de los polos de crecimiento como vía para promover el desarrollo de los territorios periféricos y atenuar la divergencia. Para impulsarlas, se suponía que el Estado debía recurrir a una política activa, basada en instrumentos discriminados territorialmente, como incentivos y desincentivos fiscales y financieros, subsidios, tarifas y precios diferenciados, controles, empresas públicas etc., dando origen a lo que Del Río \& Cuadrado (1994) han denominado como la "antigua política regional".

\section{Equilibrio}

\section{y convergencia en las previsiones neoclásicas}

El comienzo del período de auge de los modelos neoclásicos de crecimiento, en los que la tasa de crecimiento es determinada fundamentalmente por la oferta y la eficacia de los factores, puede establecerse en febrero de 1956, fecha en que Solow publicó su artículo A contribution to the theory of economic growth. El modelo propuesto entonces por este autor, concebido como respuesta y como alternativa a los de tipo keynesiano (en particular, a los de Harrod (5) y Domar), está estructurado en torno al supuesto de que "el sistema tenderá, a largo plazo, a acercarse al camino al steady state (6) y a volver a él después de cualquier perturbación” (Hahn \& Matthews, 1965: 60).

Algunas ideas e hipótesis centrales caracterizan la función de producción básica de esta propuesta y sustentan las predicciones que de allí se pueden derivar: el factor nivel tecnológico, cuya importancia se considera fundamental para el crecimiento, tiene carácter exógeno (no depende del comportamiento del ahorro y de la política económica) y viene incorporado al capital; la demanda se ajusta pasivamente a la oferta y los procesos productivos están ceñidos por retornos constantes a escala. A ello Solow agrega una "hipótesis fundamental: el rendimiento marginal del capital es decreciente", esto es, que el rendimiento de la inversión y, por lo tanto, la tasa de crecimiento del stock de capital per capita, disminuye a medida que este stock crece (7).

En este contexto, la tasa de crecimiento del producto per capita a largo plazo depende fundamentalmente de la tasa de incorporación de progreso técnico, en tanto que la acumulación de capital no produce mayor o menor crecimiento. En palabras de Solow (1987: 12-13): "la tasa de crecimiento permanente de la producción por unidad de insumo de mano de obra es independiente de la tasa de ahorro (inversión), y depende por entero de la tasa de progreso tecnológico en el sentido más amplio". 
Formalmente, el modelo de Solow posee un punto estacionario único y estable, que será alcanzado "sean cuales sean las condiciones iniciales", dado que si el progreso técnico se difunde por el mundo entero, "es posible prever que habrá convergencia de las tasas de crecimiento per capita y, aún, de los niveles de ingreso per capita" (D’Autume, 1995: 34). Esto es así, en la medida que se supone que las economías se diferencian únicamente por la relación inicial entre capital y trabajo, lo que permite alentar que en el mundo real sería esperable un crecimiento más elevado en las economías pobres que en las ricas (Sala-i-Martin, 1994: 25). En la medida que las previsiones indican que la evolución de los procesos económicos regidos por el juego de las fuerzas del mercado marchan inexorablemente hacia una mayor convergencia, la visión resultante termina siendo alentadoramente optimista.

A partir de la fecha de la publicación del trabajo de Solow, estas ideas se ubicaron como un punto de referencia obligado para las discusiones teóricas sobre el tema, donde se reconocía que “... el llamado enfoque neoclásico del análisis del crecimiento económico (...) representa el método dominante de la economía del crecimiento" (Jones, 1975: 83). Sin embargo, su influencia política sólo llegaría algunos años más tarde cuando, durante la década de los años 80, la profundización de la crisis fiscal del Estado keynesiano obligó a buscar nuevos caminos. Fue entonces cuando comenzó a hacerse evidente la creciente aceptación política de ciertas derivaciones del modelo neoclásico, ahora como fundamento de las estrategias neoliberales.

Desde ese momento sus hipótesis, explicaciones, predicciones y propuestas centrales - con frecuencia en una versión extremadamente simplificada - favorecidas por el aval de los más poderosos organismos internacionales, constituyeron los pilares de la política económica en muchos países y regiones. La nueva receta indicaba que sería posible marchar hacia una mayor convergencia, en la medida que hubiese voluntad política y capacidad para ceñirse a unas recetas simples (8): liberalización económica que permitiese restituir el libre juego de las fuerzas del mercado, consecuente reforma del Estado guiada por estrictos principios de subsidiaridad y de neutralidad, amplia apertura externa que permitiese integrar mercados a escala mundial.

Bajo el predicamento de esta receta, se asumió que para superar los desequilibrios interregionales no se requería de una politica específica a esos efectos, puesto que la operación de un mercado libre debía desembocar naturalmente en un mayor equilibrio entre territorios de desigual nivel de desarrollo y, por lo tanto, la aplicación de una política de esa naturaleza implicaría una inaceptable interferencia en ese camino. En otras palabras, la política económica debería ser concebida como un medio para restablecer y asegurar 
el libre juego del mercado, de manera de que éste pudiese conducir hacia la previsible convergencia.

\section{Crecimiento economico endogeno y retorno a la divergencia}

Contrariando las predicciones neoclásicas sobre convergencia, diversos estudios sobre el comportamiento y la evolución de la economía mundial comenzaron a proporcionar indicios que autorizaban a ponerlas en tela de juicio. De hecho, un conjunto de regularidades "típicas" - o "hechos estilizados", conforme a la terminología de Kaldor (1965) - mostraban síntomas evidentes de divergencia en los procesos observados de crecimiento: el capital fluye mayoritariamente entre los países de altos ingresos; se observa una correlación positiva entre crecimiento de largo plazo e inversión en maquinaria y equipo; existe un alto grado de asociación entre el crecimiento económico y el nivel de desarrollo científico y tecnológico de cada país; el gasto en I\&D, así como las principales innovaciones, muestran una marcada tendencia a concentrarse en los países más ricos; tanto la renta per capita como la productividad de la industria manufacturera, están intensamente relacionadas con los niveles de acumulación de capital y de mecanización; los mayores grados de desarrollo están correlacionados con una mayor productividad tanto del trabajo cuanto del capital. Al mismo tiempo que estas tendencias sugerían la persistencia de un crecimiento desigual y divergente, daban pie para cuestionar la pertinencia de las previsiones y de las prescripciones que se derivaban del modelo neoclásico.

Fue así que desde la segunda mitad de la década de los años 80 , desde el mismo corazón del pensamiento neoclásico, cobraron impulso diversos esfuerzos encaminados a formalizar modelos de crecimiento más consistentes con el cuadro caracterizado por hechos estilizados como los mencionados. En lo fundamental, desde los trabajos pioneros de Romer (1986) y Lucas (1988), comenzaron a plantearse diversos supuestos, hipótesis y factores diferentes a los considerados por la vertiente neoclásica ortodoxa. A partir de allí, se multiplicaron las contribuciones en esta dirección, destacándose, entre muchos otros, más de las posteriores de estos mismos autores, las de Rebelo, Barro, Aghion \& Howitt y Grossman \& Helpman (Sala-i-Martin, 1994; Guellec \& Ralle, 1995). Pese a que todavía no han conformado un todo enteramente coherente, estos nuevos modelos han adquirido un marcado protagonismo en los debates sobre crecimiento económico, llevando la discusión hacia tópicos diferentes a los que habían protagonizado el período anterior. 
Identificadas en su conjunto como nuevas teorias de crecimiento o modelos de crecimiento endógeno (MCE), tienen como rasgo distintivo básico su estructuración en torno a una función de producción donde la tasa de crecimiento depende básicamente del stock de tres factores: capital físico, capital humano y conocimientos (o progreso técnico), que pueden ser objeto de acumulación $y$, además, generan externalidades. Al asumir la existencia de externalidades positivas, los MCE sustituyen los supuestos neoclásicos sobre rendimientos constantes a escala y competencia perfecta, por los de rendimientos crecientes (9) y competencia imperfecta (10), con lo que sus conclusiones se alejan de la predicción de la convergencia (11).

El alcance que se asigna a cada uno de los factores considerados en la respectiva función de producción permite apreciar la especificidad del aporte de los MCE. En primer lugar, a diferencia de los planteamientos de la macroeconomía neoclásica del equilibrio, postulan la existencia de externalidades positivas asociadas a la inversión, que se traducen en retornos crecientes a escala, lo que equivale a afirmar que las que se derivan del aumento del stock de capital (incluyendo el capital humano) son de mayor magnitud que las establecidas por el modelo de Solow.

En segundo lugar, desde el modelo de Lucas (1988), los MCE enfatizan que también existen externalidades positivas asociadas al capital humano, donde el crecimiento del stock respectivo está condicionado por el volumen de recursos destinados al sector que lo produce. En esta dirección, los MCE anotan, con énfasis diferente según los autores, la importancia de los procesos de aprendizaje en la práctica (learning by doing) como un camino que permite mejorar y aumentar el stock de capital humano (12). En tercer lugar, consideran al stock de conocimientos como un factor productivo específico, resultante de una actividad económica remunerada, cuyas externalidades afectan la estructura de los mercados y, por ende, la modalidad de concurrencia. Esta forma de incorporar al progreso técnico en la función de producción del modelo, tiene como consecuencia directa la revalorización de la educación formal y de la I\&D en el proceso de acumulación de conocimientos.

En estos procesos, la empresa privada aparece como el protagonista principal de la acumulación de factores productivos y como responsable de los aumentos de productividad y, por lo tanto, de la posibilidad de un mayor crecimiento. En lo fundamental, se considera que "el desarrollo de la capacidad empresarial contribuye al aumento de la productividad y de los ingresos por habitante no solamente por medio de su capacidad creativa sino también a través de la imitación e incorporación de conocimiento previamente 
desarrollado" (Mujica, 1991: 27). De donde, el nivel empresarial en términos de capacidad innovadora y de eficiencia en la gestión puede considerarse como un indicador del potencial de crecimiento alcanzado.

Conforme al razonamiento general que caracteriza a estos modelos, la tasa de acumulación de los factores productivos (en especial, la de cambio técnico) y, por lo tanto, la tasa de crecimiento, antes que de factores exógenos, dependen básicamente de las decisiones adoptadas en un determinado y concreto entorno económico. En otras palabras, el crecimiento a largo plazo es un fenómeno económico endógeno, producto del hecho de que las inversiones respectivas - en especial las destinadas a la generación de capital humano y de progreso técnico - son efectuadas por actores económicos motivados por la ganancia (13). En síntesis, el nivel de ingreso a largo plazo de un determinado territorio estaria determinado por la acumulación de capital físico, capital humano y conocimientos, donde los niveles respectivos pueden considerarse como variables endógenas, determinados por decisiones de ahorro e inversión motivadas por expectativas de ganancia.

\section{Significado y alcance \\ de los nuevos modelos de crecimiento}

Los nuevos modelos de crecimiento han recibido diversas críticas y objeciones, donde se ha cuestionado tanto la originalidad de sus contribuciones, como algunos de sus desarrollos sustantivos. Así, por una parte, se ha anotado el hecho de que muchos de sus aportes no significan mucho más que la actualización y formalización de diversos aspectos que habían estado presentes en la literatura sobre crecimiento económico desde bastante tiempo atrás. En particular, se ha señalado que el énfasis en el crecimiento endógeno no constituye una contribución novedosa, por cuanto esta idea ya había sido incorporada en modelos como los de Arrow, Harrod, Kaldor, Robinson y Schumpeter, entre otros. En esta dirección, Nelson (1997: 2;15) afirma que "estos modelos adoptan en forma estilizada varios aportes sobre avance técnico que por muchos años habían sido bien documentados por investigadores empíricos" y que "los fenómenos incorporados en los nuevos modelos formales, y omitidos en muchos de los viejos, escasamente representan conocimientos o ideas novedosos". Todo ello lleva a Neri \& Salvadori (1995) a afirmar que el contenido esencial de estos modelos no sería más que "viejo vino en nuevos odres".

Por otra parte, tanto desde la propia perspectiva neoclásica como desde la de otras vertientes de la teoría económica, se han cuestionado algunos aspectos sustantivos de la nueva formulación. Así, por una parte, Solow (1994) 
objeta el abandono del supuesto de los rendimientos decrecientes del capital y critica enfáticamente la hipótesis de rendimientos constantes. A su vez, desde un punto de vista diferente - el evolucionista -, se han anotado como importantes limitaciones de estos modelos para una mejor aproximación a la realidad, el que sus desarrollos continúen apegados a los cánones de la teoría del equilibrio y, por otra parte, el que consideren solamente las fuentes o determinantes inmediatos del crecimiento económico, dejando de lado los factores que están por detrás de los mismos. A este respecto, se critica la omisión de aspectos tales como los relativos a la generación, verificación y avance de la tecnología, la naturaleza y el rol de las instituciones que establecen el medio en el cual operan las firmas y, en particular, el tratamiento extremadamente simplificado que los MCE hacen sobre éstas (Nelson, 1997: 19).

Por encima de este conjunto de reparos, es posible reconocer que el cambio de enfoque impulsado por estos modelos resulta más consistente con los hechos estilizados y, por lo tanto, que la explicación que suministran vis-a-pis la realidad observable, resulta más convincente que la ofrecida por los modelos neoclásicos ortodoxos. En particular, al aceptar la existencia de competencia imperfecta y oligopólica y de divergencia económica, los MCE están en condiciones de admitir y explicar la tendencia a la acumulación concentrada de capital físico, capital humano y conocimientos, tanto en el plano económico como en el territorial.

$\mathrm{Al}$ mismo tiempo, al considerar que la acumulación y el crecimiento son fenómenos económicos endógenos resultantes de un conjunto de decisiones de ahorro-inversión adoptadas en función de la ganancia, los MCE orientan su análisis hacia las condiciones y los factores que contribuyen a mejorar la valorización privada del capital en el ámbito económico en que esas decisiones se hacen efectivas. Esto, por cuanto se asume que la tasa de crecimiento en un específico ámbito territorial depende fundamentalmente de la percepción que tienen los actores que adoptan las decisiones de inversión (o no inversión) sobre las condiciones que allí prevalecen para la valorización de sus capitales. Por lo tanto, habida cuenta de los diversos aspectos y factores que incorporan y privilegian, los MCE permiten un análisis más realista sobre la forma en que se produce el crecimiento (y, eventualmente, el desarrollo) en los escenarios emergentes de la reestructuración y la globalización.

\section{Politicas publicas para el crecimiento endogeno}

¿Qué consecuencias para la política pueden inferirse de estos modelos? Conforme a los atributos del entorno que los MCE consideran como más favorable para el crecimiento, en una primera aproximación, se puede inferir 
que la politica económica debería tener como función principal contribuir a generar un ambiente macroeconómico propicio para la mejor valorización privada del capital. En este sentido, Sala-i-Martín (1994: 7) precisa que la aparición de gobiernos que garanticen los derechos de propiedad física e intelectual, que regulen el sector financiero y exterior y eliminen las distorsiones, y que mantengan un marco legal garante del orden es deseable. El gobierno, por lo tanto, juega un papel importante en la determinación de la tasa de crecimiento a largo plazo.

Conforme a esta aproximación general, Guellec y Ralle (1995: 94 y ss.) señalan que más allá de la heterogeneidad de las propuestas de esta corriente, se pueden identificar en ellos dos grandes campos para una intervención pública orientada a asegurar el mejor despliegue de las fuerzas del mercado: la gestión de las externalidades y la provisión de ciertos bienes públicos. Al aceptar la presencia de externalidades vinculadas a la acumulación de capital físico, capital humano y conocimientos y, por lo tanto, de concurrencia imperfecta, los MCE reconocen que existen defectos en la coordinación entre los actores privados y que esto obstaculiza la obtención de un óptimo social; en estas circunstancias, aceptan ciertas formas de intervención pública orientada a modificar el entorno institucional en el que se generan dichas externalidades, buscando establecer las condiciones para mejorar la coordinación de los actores involucrados. Por otra parte, al considerar que los bienes públicos pueden tener una incidencia positiva en la productividad privada, algunos autores de esta corriente reconocen que una parte del capital total debe ser público.

En cualquier caso, estas áreas de intervención están encuadradas siempre por la idea de que la política económica tiene como misión favorecer la creación de un ambiente estimulante para la inversión, pero sin alterar el juego de las fuerzas del mercado. De esta manera, si bien los MCE aceptan un papel algo más activo del Estado y de la política económica que los modelos neoclásicos ortodoxos, en modo alguno ello puede interpretarse como una suerte de retorno al intervencionismo de la época keynesiana.

En este contexto general, cuando se aborda el tema de las políticas para el caso de territorios nacionales o subnaciones específicos, estos modelos introducen algunos aspectos específicos que importa destacar. Así, en tanto asumen que las decisiones de inversión que permiten incrementar los stocks de capital físico, humano y técnico tienen carácter endógeno, los MCE revalorizan un aspecto desdeñado por los modelos neoclásicos ortodoxos, cual es la situación inicial de cada territorio, en tanto condicionante de su potencial endógeno. Este concepto, que puede considerarse como equivalen- 
te al de fertilidad propuesto Kampetter (1995), indica básicamente la mayor o menor aptitud de cada territorio para que alli puedan germinar nuevas inversiones en capital físico y humano y en conocimientos; por lo tanto, su capacidad para continuar creciendo y avanzando hacia niveles superiores de desarrollo en el ámbito de la actual dinámica globalizada. De hecho, estos conceptos se sitúan como el punto de partida para la mayor parte de las políticas de crecimiento para territorios específicos.

Al postular que el potencial endógeno es lo que condiciona básicamente las perspectivas de crecimiento regional, toda vez que se trata de definir una política para un territorio en particular, se considera necesario ante todo evaluar la potencialidad de su situación inicial e identificar las condiciones y los mecanismos que podrían favorecer en mayor grado las inversiones requeridas para que allí se produzca un incremento efectivo de los stocks de los factores acumulables. Estos antecedentes aparecen como imprescindibles para precisar los lineamientos de una gestión orientada a establecer o fortalecer dichas condiciones y mecanismos con el objetivo de incrementar la atractividad $^{1}$ de ese lugar $y$, consecuentemente, de activar su potencial endógeno. En esta dirección, como ya se ha señalado, aparece como propósito prioritario de la política respectiva la configuración de un ambiente económico, social y político ventajoso para la valorización privada del capital, de manera que el mismo resulte atractivo, especialmente para las empresas más innovadoras y competitivas, cuya localización en ese ámbito se estima fundamental para que allí puedan desencadenarse procesos sostenidos de crecimiento endógeno.

Dados sus supuestos básicos, este nuevo enfoque de gestión territorial plantea que debe ser cada comunidad territorial (nacional o subnacional) la que tome la iniciativa y adopte las medidas requeridas para estimular la activación del respectivo potencial endógeno, por lo que el nuevo paradigma implica un desplazamiento de la propuesta del crecimiento desde arriba hacia una de crecimiento desde abajo. Si bien esta nueva forma de abordar las estrategias de crecimiento se considera aplicable en cualquier nivel territorial - nacional, regional y local - (15), ellas han adquirido su expresión más destacada en las propuestas sobre desarrollo local, las que han adquirido un amplio predicamento en los últimos años.

En todo caso, los criterios básicos y las propuestas concretas que habitualmente se encuentran en los planteos de la denominada nueva política regional o de crecimiento endógeno (Cappellin, 1992; Garófoli, 1995; Vázquez Barquero, 1996) muestran claramente la influencia que sobre ella han tenido los aportes de los MCE (16). Aspectos tales como desarrollo del 
capital humano, creación de condiciones para el aprendizaje en la práctica, aumento de las capacidades empresariales y de innovación, intensificación de los esfuerzos por estimular la especialización regional, profundización de la inserción externa, entre otros, aparecen reiteradamente en el discurso del nuevo paradigma. Con estos fundamentos, éste ha adquirido una gran popularidad, aún cuando cabe observar que en muchos casos el alcance que allí se otorga al concepto de crecimiento endógeno tiende a simplificar el significado que el mismo tiene en la función de producción y en las explicaciones de los MCE. En todo caso, estas propuestas han comenzado a tener una ascendente influencia como receta para enfrentar los problemas de crecimiento en el caso de territorios nacionales o subnacionales de menor desarrollo relativo.

\section{Crecimiento endogeno y territorios de menor desarrollo}

Más allá de las objeciones de que han sido objeto, también puede reconocerse que los MCE suministran un marco teórico útil para plantear el análisis sobre la posibilidad de impulsar un efectivo crecimiento en el caso de territorios menos desarrollados, de forma de atenuar la brecha de las desigualdades interregionales y de avanzar hacia una mayor convergencia. Como ya se ha señalado, según estos modelos, esta posibilidad estaría condicionada básicamente por el nivel de acumulación (en equipos, en infraestructuras, en educación, en conocimientos) realizada a lo largo de la respectiva historia productiva de cada territorio, así como por algún tipo de ventaja comparativa vis-a-pis el mercado global, generalmente asociada a recursos naturales. De hecho, esto presupone que la desigual acumulación inicial entre distintos territorios, puede constituirse en un factor adverso para los menos desarrollados vis-a-vis los más desarrollados, sentando las bases para la retroalimentación de una evolución divergente.

Si se acepta que dicho nivel inicial de acumulación de capital físico, capital humano y conocimientos en un determinado territorio juega un papel crucial en cuanto a sus perspectivas de crecimiento, resulta lógico preguntarse hasta dónde, una política orientada a activar el potencial endógeno puede modificar las diferencias interregionales en la acumulación inicial de los factores que explican el crecimiento y, de esta forma, atenuar la brecha en las disparidades de ingresos existente entre territorios de desigual nivel de desarrollo. Con el propósito de esbozar una respuesta al respecto, importa analizar por una parte la situación que afecta a los procesos de acumulación de capital físico y, por otra parte, de capital humano y conocimientos. 
En lo que concierne a la posibilidad de incrementar la acumulación de capital físico, la principal dificultad que enfrentan los territorios menos desarrollados radica en el hecho de que en virtud de su menor potencial endógeno (o fertilidad) inicial, por lo general resultan menos atractivos para unos capitales que, por esta razón, solamente tienden a orientarse marginalmente hacia ellos. El hecho es que habitualmente los propietarios del capital, al momento de adoptar sus decisiones de inversión-acumulación, se guían por un conjunto de informaciones e indicaciones disponibles sobre la situación de los distintos territorios en que podrían realizar sus inversiones.

Un buen ejemplo de este tipo de indicaciones es el que suministran las evaluaciones riesgo-país, cuyo propósito fundamental es justamente determinar la mayor o menor atractividad para la inversión ofrecida por distintos ámbitos (generalmente naciones o empresas) y establecer sus respectivas ubicaciones relativas (17) (Marois, 1990). En la medida que los territorios de menor desarrollo aparecen en general pobremente calificados en los rankings respectivos es lógico que los inversores privados, al momento de adoptar sus decisiones sobre la localización de sus inversiones, se orienten hacia lugares mejor calificados. En particular, estas indicaciones inciden decisivamente en el caso de las empresas más modernas e innovadoras (18).

La realidad observable suministra abundante evidencia empírica sobre la marcada preferencia de los inversores privados por los territorios de mayor desarrollo relativo (19), pese a que allí los salarios y los impuestos sean más altos y las regulaciones más estrictas; lo cual, como es obvio, opera en desmedro de los menos desarrollados, aún cuando allí los salarios y los impuestos sean más bajos y las regulaciones más suaves (Kampetter, 1995). En estas circunstancias, una política orientada a establecer las condiciones para promover el crecimiento endógeno de un territorio de menor desarrollo debe proponerse ante todo mejorar una atractividad que no solamente depende de aquellos factores que habitualmente favorecen a estos territorios (como, por ejemplo, menor costo de la mano de obra o disponibilidad de recursos naturales), sino también de otros en los que normalmente no presentan ventajas comparativas ( tales como calificación de la fuerza de trabajo, calidad y densidad del tejido industrial, disponibilidad de modernas infraestructuras de transportes y telecomunicaciones, existencia de mercados de capitales consolidados etc.).

Si esto es así, ¿̇en qué medida es posible cambiar la atractividad de un territorio de manera de capacitarlo para captar mayores volúmenes de capital? A este respecto, los instrumentos de política aceptables según las reglas 
de juego dominantes en el marco de la globalización no parecen suficientemente efectivos como para cambiar en forma significativa la dirección de unos capitales que, como ya se ha señalado, se orientan predominantemente hacia los territorios más atractivos, dada su mayor fertilidad relativa inicial o su mejor calificación riesgo-país. De tal forma, una gestión endógena orientada a incrementar los niveles de acumulación en un territorio de menor desarrollo relativo (nacional o subnacional) dispone de escasa fuerza para lograr estos objetivos.

Así lo muestran algunas experiencias desarrolladas con el propósito de atraer inversiones hacia nuevas actividades productivas en territorios periféricos. En este sentido, numerosos gobiernos nacionales, regionales y/o locales han estado otorgando distintos tipos de ventajas a las empresas a través de medidas como subvenciones, terrenos gratuitos, exoneraciones fiscales etc., en lo que Glickman \& Woodward (1989: 275) han calificado como una "frenética lucha por las migajas", puesto que no consiguen alterar significativamente las ya señaladas tendencias dominantes; por lo tanto, los montos de inversión que efectivamente se logran desviar son, por lo general, poco relevantes. Además, con la aplicación de este tipo de política muchas veces se termina perdiendo más de lo que se gana, en la medida que" ... podría tratarse de lo que los especialistas llaman la carrera hacia abajo: una competencia contraproducente por atraer a empresas que ofrecen pocas ventajas para la región a cambio de todas las que reciben (Moffett, 1997).

En consecuencia, todo indica que los esfuerzos por captar capitales externos, que generalmente aparecen asociados a las políticas de crecimiento endógeno para territorios menos desarrollados, difícilmente pueden lograr revertir las desigualdades iniciales y solamente como excepción logran aproximarse a los objetivos previamente establecidos. Lo que tiende a confirmar la tesis de que son muy pocos los territorios embarcados en este juego pueden resultar ganadores.

Por su parte, un análisis a fondo sobre la posibilidad de atenuar la brecha en términos de acumulación de capital humano y de conocimientos existente entre territorios de desigual nivel de desarrollo debería considerar las condiciones en que se desenvuelven los procesos de reproducción de las estructuras sociales y las posibilidades de alterarlas. Esto llevaría a incursionar en un área fundamental de investigación toda vez que se plantea el tema de las desigualdades sociales y culturales, aún cuando todavía poco explorada, cuyo tratamiento a fondo excede los límites de este trabajo.

Desde esta perspectiva habría de partir del hecho de que el espacio social en territorios de distinto nivel de desarrollo muestra sustantivas dife- 
rencias en la dotación capital económico y de capital cultural (20) y de que en cada uno de estos niveles operan mecanismos de reproducción social "que hacen que el capital vaya al capital y que la estructura social tienda a perpetuarse - no sin antes experimentar unas deformaciones más o menos importantes" (Bourdieu, 1994: 33). Estos mecanismos operan al interior de cada territorio y, por lo tanto, en las relaciones entre territorios de desigual nivel de desarrollo. Esto es, en la medida que se reproducen las diferencias al interior de un territorio de menor desarrollo, las desigualdades entre éste y otros territorios de mayor desarrollo también tiende a persistir.

¿Cuáles son los mecanismos por los que se reproduce la estructura del capital cultural en un determinado espacio social? Según Bourdieu (1994: 33) ello "se lleva a cabo en la relación de las estrategias de las familias y la lógica específica de la institución escolar", en la medida que las familias se mueven según "una tendencia a perpetuar su ser social, con todos sus poderes y privilegios" y, a su vez, el sistema escolar "mediante toda una serie de operaciones de selección, separa a los poseedores de capital cultural heredado de los que carecen de él", con lo que se "mantiene el orden preexistente, es decir la separación entre los alumnos dotados de cantidades desiguales de capital cultural".

En una dinámica que funciona con esta lógica, cualquier intento de reducir las diferencias iniciales haría necesario modificar las estrategias familiares y el funcionamiento del sistema escolar. Sin embargo, dado que ambos, familias y escuela, son resultado de transformaciones de carácter estructural producidas a lo largo de un prolongado período de la historia de cada espacio social, su resistencia al cambio es muy fuerte, lo que redunda en que cualquier intento de alterarlas solamente pueda cristalizar en el largo plazo. Aún desde una perspectiva más estrecha, la estrictamente económica, los propios MCE reconocen que ciertas habilidades y formas específicas de conocimiento sólo pueden adquirirse a través del tiempo, como resultado de la información que proporcionan los mercados y la experiencia acumulada en el propio proceso productivo (Mujica, 1991: 25).

Además, estos procesos deben cumplirse en situación desventajosa con respecto a los territorios de mayor desarrollo relativo, por cuanto el objetivo de incrementar el stock de capital humano en un territorio de menor desarrollo deberá enfrentar el obstáculo de que justamente en virtud de esa condición, la situación inicial se caracteriza por una baja dotación de este recurso, cuando para poder aumentarlo se requeriría de importantes stocks del mismo. El hecho de que a menor dotación inicial de capital humano, menor posibilidad de incrementarlo, está en la base de la operación de un 
poderoso mecanismo de retroalimentación, difícil de neutralizar. A ello debe agregarse que para mejorar el nivel de formación de los habitantes de un territorio rezagado, se necesita contar con recursos económicos para educación mayores que los que requiere uno más desarrollado y, sin embargo, justamente debido a su menor desarrollo relativo, difícilmente podrá siquiera equipararlos.

Cuando, en particular, se considera la cuestión de la brecha existente en materia de acumulación de conocimientos tecnológicos entre territorios de diverso nivel de desarrollo, debe tenerse en cuenta que, como destaca la teoría evolucionista, "generalmente la probabilidad de realizar avances tecnológicos en firmas, organizaciones y aún naciones es función del nivel tecnológico alcanzado por ellas. (...) Las asimetrías o brechas tecnológicas entre firmas - y naciones - surgen como una consecuencia natural de estas tendencias" (López, 1996: 118). Vale decir, la situación tecnológica inicial conforma un importante handicap para el avance tecnológico para los territorios rezagados en este terreno (21), lo que además se agrava por el hecho de dada la tendencia dominante de los flujos de capital, el monto de recursos que tanto las empresas como el sector público pueden asignar a la generación de conocimientos en ellos resulta siempre inferior al disponible en los más avanzados.

Pero aún en las circunstancias más favorables, cabría preguntarse: ¿en qué medida el cambio tecnológico en un territorio periférico podría desarrollarse en forma compatible con la necesidad de mejorar la competitividad, aumentar el empleo y atenuar las desigualdades entre el ingreso de los distintos tipos de trabajadores? En esta dirección, el principal peligro que parece estar al acecho a medida que avanzan los procesos de modernización tecnológica es la tendencia hacia la polarización de la estratificación laboral; en efecto, en todo el mundo la modernización tecnológica en contextos desregulados y flexibilizados parece estar llevando a una polarización de la estructura del empleo entre ocupaciones de muy elevada y muy baja productividad y remuneraciones, lo que redunda en la conformación de mercados de trabajo extremadamente dualizados.

En este cuadro general, el análisis de procesos concretos de crecimiento en el ámbito de una economía en progresivo proceso de globalización, permite comprobar que muchas de las esperanzas que se habían cifrado en un porvenir más alentador para los territorios periféricos, hasta ahora no han sido refrendadas, tanto en el caso de los países desarrollados como, especialmente, en los que se ubican en la periferia capitalista. Así, evaluaciones realizadas desde la perspectiva de territorios de menor desarrollo en el ámbito 
de la Comunidad Europea, tienden a confirmar estas aprehensiones (22). Obviamente, estas tendencias son mucho más acentuadas para el caso de las regiones rezagadas de los países de la periferia capitalista, donde operan los mecanismos arriba esbozados. Frente a este panorama, cobra renovada vigencia una conclusión esbozada hace más de veinte años por Gilbert \& Goodman (1976: 86), en el sentido de que "una comparación entre los niveles de disparidad regional en los países de menor desarrollo hoy día y la que caracterizó a los países desarrollados en su período de máxima divergencia no alienta esperanzas de una convergencia final en los ingresos regionales".

En lo fundamental, el análisis realizado a partir de los supuestos y las hipótesis de los MCE suministra un razonable respaldo a los argumentos en favor de la tesis de la divergencia. En otras palabras, tanto los nuevos enfoques teóricos como la evidencia empírica disponible, indican que la convergencia interregional no aparece como una tendencia asociada a la propagación de una economía capitalista globalizada. De hecho, la presencia de externalidades y rendimientos crecientes permite explicar la tendencia a la localización de la cabeza de los principales procesos productivos - y, en especial, de la parte más moderna e innovadora de la industria y del terciario avanzado - en determinados centros desde donde se ejerce el comando de la vida económica a escala internacional, nacional y regional, en coexistencia con un sinnúmero de territorios periféricos que cumplen actividades marginales con limitadas posibilidades de revertir su situación.

En ese cuadro, donde el avance de la globalización ha favorecido la expansión de un número creciente de grandes empresas multinacionales que marcan el rumbo del proceso de acumulación en una economía-mundo que ha acentuado su carácter no concurrencial, no parecen haber perdido vigencia las tendencias acumulativas de diferenciación interregional y de causación circular sistematizadas varias décadas atrás. Bajo esa dinámica, si bien no es posible afirmar que están cerrados los caminos para que algunos territorios menos desarrollados puedan iniciar procesos de crecimiento sostenido que les permitan mejorar su situación futura, no parecen suficientemente justificados ciertos augurios sobre una generalización de la tendencia hacia la convergencia.

Notas

1 Para este análisis, resulta útil la distinción propuesta por Nelson (1997: 6) entre teorías apreciativas y teorías formalizadas, donde las primeras están expresadas principalmente de forma verbalizada, en tanto que las segundas se 
presentan como estructuras abstractas establecidas para permitir explorar, encontrar y verificar las conexiones lógicas propuestas.

2 En una exhaustiva revisión de las ideas dominantes en el campo de teorías del crecimiento regional en las últimas décadas, Cuadrado Roura (1995) propone una apertura más amplia que la contemplada en este trabajo, pero cuyos diferentes componentes podrían encuadrarse, en mayor o menor grado, en las tres vertientes básicas que aquí se discuten.

3 Quizás a estas vertientes teóricas cabría agregar la del evolucionismo, que se ha ido configurando con contribuciones como las de Nelson, Winter, Dosi, Freeman \& Lundvall, entre otros. En todo caso, esta vertiente aún no ha cristalizado en un cuerpo teórico unificado, ni en modelos formalizados equivalentes y su propuestas para la definición de políticas es todavía ambiguo y fragmentario. Véase una completa revisión de los aportes del evolucionismo en López (1996).

4 En el ámbito latinoamericano, estas ideas tuvieron una fuerte repercusión, que cristalizó fundamentalmente en el diagnóstico y en las propuestas normativas de la Cepal, donde los aportes de Prebisch y de Furtado marcan los hitos centrales.

5 En su "Discurso de aceptación del Premio Nobel" pronunciado en 1987, Sólow reconoce su insatisfacción con los planteos de Harrod sobre el desequilibrio en los procesos de crecimiento: "en particular, las obras de Harrod rebosaban de aseveraciones incompletamente elaboradas en el sentido de que el crecimiento sostenido era, en todo caso, una especie de equilibrio muy inestable: todo pequeño alejamiento del equilibrio se acentuaría indefinidamente por un proceso que parecía depender sobre todo de vagas generalizaciones acerca del comportamiento empresarial" (Solow, 1987: 10).

6 La expresión steady state no tiene un equivalente adecuado en lengua castellana, donde generalmente se la ha traducido como estado estacionario o estado continиo. La misma hace referencia a un estado, o una trayectoria, donde la tasa de crecimiento de todas las variables involucradas permanecen constantes a lo largo del tiempo.

7 En este último punto radica uno de los aspectos centrales de las formulaciones neoclásicas ortodoxas y marca una de las principales diferencias entre éstas y los nuevos modelos neoclásicos de crecimiento. A este respecto, Solow reconoce que “... las teorías más tradicionales plantean que la mayor inversión suscita una aceleración transitoria del crecimiento de la productividad a un nivel sostenidamente más alto; la teoría más reciente sugiere que la mayor inversión, sobre todo en capital humano y en tecnología, puede llevar a una tasa de expansión permanentemente más elevada" (Solow, 1993: 31; destacado nuestro).

8 Estas recetas, que ganaron un fuerte predicamento político desde fines de la década de los años 70, responden en mayor grado a las ideas de los economis- 
tas neoliberales vinculados a la Universidad de Chicago, como Hayek, Friedmann, Becker \& Schultz, entre otros, que a Solow, cuyas propuestas sobre política económica contemplan un papel más activo del Estado.

9 Como conclusión de un estudio sobre varios modelos de crecimiento endógeno, Kurz \& Salvadori (1995:6) cuestionan la pertinencia de esta afirmación, al señalar que "se sostiene con frecuencia que la novedad original de los nuevos modelos del crecimiento es que ellos funcionan con retornos crecientes de escala. Un análisis a fondo muestra que este no es el caso. Lo que realmente ocurre es que los retornos a escala con respecto a los factores acumulables son cuando menos constantes".

10 En la misma dirección, los nuevos modelos del comercio internacional consideran que gran parte del comercio es el resultado de una especialización arbitraria basada en la existencia de rendimientos crecientes, en lugar de ser un esfuerzo consciente de aprovecharse de diferencias exógenas o recursos o productividad (Krugman, 1992: 13).

11 Esta predicción no es compartida por todos los autores vinculados a los MCE. En particular, Lucas "sostiene que a futuro se irán sucediendo los milagros económicos en los países que ahora son pobres, que los niveles de ingreso per capita de los países convergerán y que la dispersión actual se hará mucho menos pronunciada" (Vergara, 1997: 132).

12 Ultimamente Lucas ha modificado su opinión sobre la importancia de los medios que contribuyen a incrementar el capital humano, destacando el papel del learning by doing por sobre el de la educación formal (Lucas, 1997; Vergara, 1997).

13 En particular, Romer enfatiza que el crecimiento en este modelo es conducido por el cambio tecnológico que surge de las decisiones intencionales de inversión hechas por agentes maximizadores de ganancia (Romer,1990: 71).

14 Obviamente existe una estrecha relación entre los conceptos de potencial endógeno, fertilidad territorial y atractividad. Con respecto a este último, Marconnet \& Fremeaux (1996: 70) precisan que "la atractividad de un territorio depende de múltiples factores: costo y calidad de la mano de obra, pero también calidad del tejido industrial local (para la subcontratación), calidad de las infraestructuras de transportes y comunicaciones, apertura del mercado de capitales, ayudas políticas a la inversión".

15 En todo caso, cuando se analizan las condiciones para el crecimiento de un territorio subnacional (regional o local), no se puede dejar de considerar que su atractividad, ante todo está acotada críticamente por la de su entorno nacional, lo que significa que resulta mucho más difícil impulsar una gestión endógena exitosa a este nivel, cuando es escasa la del respectivo ámbito nacional. Como ha enfatizado Porter, "las diferencias a escala nacional en estructuras económicas, 
valores, culturas instituciones e historias contribuyen profundamente al éxito competitivo. El papel de la nación parece ser tan fuerte como antes o incluso más fuerte que nunca" (Porter, 1990: 45).

16 En muchas de estas nuevas propuestas se entrecruzan conceptos y desarrollos procedentes de los MCE con los de la vertiente evolucionista, que también destaca el carácter endógeno de los procesos de crecimiento y cambio.

17 Por ejemplo, la clasificación de riesgo-país de Standard \& Poor's, evalúa la situación de cada país en función de su riesgo político y de su riesgo económico. Se considera que el riesgo político depende del sistema político, del ambiente social y de las relaciones internacionales. A su vez, el riesgo económico depende de la posición financiera externa, de la flexibilidad de la balanza de pagos, de la estructura y el crecimiento económico, de la conducción económica y de las perspectivas económicas. (Standard \& Poor's, Emerging Markets, marzo 1995).

18 Como afirma Vázquez Barquero (1997: 9), "las empresas innovadoras se sienten atraídas por localizaciones que tienen recursos e infraestructuras de calidad y capacidad innovadora, cuyo sistema local de empresas es susceptible de generar economías externas de escala y de producir bienes y servicios, en condiciones de competencia creciente, y cuyo marco institucional favorece el desarrollo de un clima empresarial proclive a la cooperación y la competitividad". Esto es, por territorios dotados de mayor potencial endógeno y, por ende, de una también mayor atractividad.

19 Múltiples testimonios documentan esta tendencia. Al respecto, Marconnet \& Frémeaux (1996: 68) señalan que a la inversa de la idea recibida según la cual la inversión internacional va en primer lugar hacia los países en desarrollo, la IED se ha concentrado en el Norte: $80 \%$ han sido realizadas en la zona de la OECD durante los años 80 , contra $20 \%$ en los países en desarrollo. Nada muy sorprendente: es en el seno de la OECD donde se encuentran los mercados más importantes y es allí donde están los recursos necesarios para producir. Por su parte, Krugman (1996: 61) señalaba que "el auge de inversiones en los mercados emergentes ha reducido desde 1990 el stock de capital del mundo avanzado en sólo aproximadamente un 0,5 por 100 de lo que de otra forma habría sido".

20 El concepto más amplio y complejo de capital cultural utilizado por Bourdieu, que engloba los conceptos de capital humano y de conocimientos, resulta de utilidad para plantear este punto.

21 Como afirma Dosi, “... las apuestas innovadoras hunden sus raíces en el pasado, reflejan el desarrollo pretérito de la empresa y de la economía nacional y tienden a seguir la dirección de los trabajos emprendidos con anterioridad. Los recursos disponibles para abordar la siguiente ronda de problemas técnicos refleja lo que ha sucedido, es decir, la tecnología tiene historia". (Dosi, 1991: 185). 
22 Así, por ejemplo, al realizar un balance desde la perspectiva de la economía andaluza, Delgado Cabeza (1996: 233) concluye que lo que ahora se puede observar es un desarrollo desigual en la Europa del Mercado Unico caracterizado por la contradicción entre espacios privilegiados, grandes áreas de innovación en las que se sitúa la capacidad para generar y gestionar una densa malla de flujos financieros y de información, y otros al margen de estos procesos, aunque crecientemente condicionados por ellos, en clara posición de fuera de juego. Las conclusiones de Hadjimichalis (1994), desde la perspectiva de otras regiones del sur de Europa, apuntan en la misma dirección.

\section{Referências bibliográficas}

ASSIDON, Elsa. Les théories économiques du developpement. Paris, La Decouverte, 1992.

BEAUD, Michel \& DOSTALER, Gilles. La pensée économique depuis Keynes. Paris, Editions du Seuil, 1993 [edición abreviada en 1996].

BOURDIEU, Pierre. Razones prácticas. Sobre la teoría de la acción. Barcelona, Editorial Anagrama, 1997.

CAPPELLIN, Ricardo Los nuevos centros de gravedad del desarrollo regional en la Europa de los 90. Malaga, Revista de Estudios Regionales, n. 33, 1992.

CUADRADO ROURA, Juan Ramón. Planteamientos y teorías dominantes sobre el crecimiento regional en Europa en las cuatro últimas décadas. Santiago de Chile, Revista EURE, n. 63, jun. 1995.

D'AUTUME, Antoine. Les nouvelles théories de la croissance. Paris, Cahiers Français, n. 272, jul./set. 1995.

DELGADO CABEZA, Manuel. Integración y reestructuración desde la periferia europea. Malaga, Revista de Estudios Regionales, n. 44, ene./abr. 1996.

DEL RIO, Clemente \& CUADRADO ROURA, Juan Ramón. La importancia de los servicios a la producción en la nueva política regional. En José Luis Curbelo y otros. Territorios en transformación. Análisis y propuestas. Madrid, Fondo Europeo de Desarrollo Regional/Consejo Superior de Investigaciones Científicas, 1994.

DE MATTOS, Carlos A. Modelos de crecimiento económico endógeno y divergencia interregional, ¿nuevos caminos para la gestión regional?. Documentos, Serie Azul/11, Santiago de Chile, Instituto de Estudios Urbanos, dic. 1996.

DOSI, Giovanni. Una reconsideración de las condiciones y los modelos de desarrollo. Una perspectiva 'evolucionista' de la innovación, el comercio y el crecimiento. Madrid, Pensamiento Iberoamericano, n. 20, jul./dic. 1991. 
GAROFOLI, Gioacchino. Desarrollo económico, organización de la producción y territorio. En: A. Vázquez Barquero \& G. Garofoli (eds.). Desarrollo económico local en Europa. Madrid, Colegio de Economistas de Madrid, 1995.

GILBERT, Alan G. \& GOODMAN, David E. Desigualdades regionales de ingreso y desarrollo económico: un enfoque crítico. Santiago de Chile, Revista EURE, n. 13, jun. 1976.

GLICKMAN, Norman \& WOODWARD, Douglas P. Los nuevos competidores. Los inversores extranjeros cambian la economía norteamericana. Barcelona, Editorial Gedisa, 1994.

GUELLEC, Dominique \& RALLE, Pierre. Les nouvelles théories de la croissance. Paris, La Decouverte, 1995.

HAHN, F.H. \& MATTHEWS, R.C.O. La teoría del crecimiento económico: una visión panorámica. En: F.H. Hahn et alli (eds.). Panoramas contemporáneos de la teoría económica, v. II. Madrid, Alianza Editorial, 1970.

HADJIMICHALIS, Costis. Las regiones periféricas en Europa en transición: una perspectiva desde el sur. Santiago de Chile, Revista EURE, n. 59, mar. 1994.

HIRSCHMAN, Albert. O. The strategy of economic development. New Haven, Yale University Press, 1958.

JONES, Hywell. Introducción a las teorías modernas del crecimiento económico, $2^{2}$ ed. Barcelona, Antoni Bosch Editor, 1988.

KALDOR, Nicholas. The case for regional policies. Scottish Journal of Political Economy, v. XVII, n. 3, nov. 1970.

Capital accumulation and economic growth. En: F.A. Lutz \& D.C. Hague (eds.). The theory of capital. Londres, International Economic Association/MacMillan, 1965.

KAMPPETER, Werner. Fertilidad nacional, Estado-nación y sistema económico mundial. Caracas, Nueva Sociedad, n. 137, mayo/jun. 1995.

KRUGMAN, Paul. El internacionalismo "moderno". La economía internacional y las mentiras de la competitividad. Barcelona, Grijalbo Mondadori, 1997.

Geografía y comercio. Barcelona, Antoni Bosch (ed.), 1992.

KURZ D., Heinz \& SALVADORI, Neri. The 'new' growth theory: old wine in new goatskins. Postkeynesian thought page. World Wide Web, 1995.

LUCAS, Robert E. Conversación con Robert E. Lucas, Jr. Santiago de Chile, Estudios Públicos, n. 66, otoño 1997. 
LUCAS, Robert E. On the mechanics of economic development. Journal of Monetary Economics, n. 22, jul. 1988.

MARCONNET, Isabelle \& FREMEAUX, Philippe. La nouvelle geographie de l'investissement international. Paris, Alternatives Economiques, n. 133, ene. 1996.

MAROIS, Bernard. Le risque-pays. Paris, Presses Universitaires de France, 1990.

MOFFETT, Matt (The Wall Street Journal). Los estados de la región más pobre de Brasil ofrecen más de lo que reciben con tal de asegurarse negocios. Santiago de Chile, El Mercurio, 29 ago. 1997.

MUJICA, Patricio. Nuevos enfoques en la teoría del crecimiento económico: una evaluación. Documento de Trabajo n. 2. Santiago de Chile, Cepal, División de Desarrollo Económico, 1991.

MYRDAL, Gunnar. Teoría económica y regiones subdesarrolladas. México, Fondo de Cultura Económica, 1959.

NELSON, Richard. How new is new growth theory? A different point of view. Challenge, v. 44, n. 5, set./oct. 1997.

PERROUX, François. Note sur la notion de pole de croissance. Paris, Economie Appliquée, t. VIII, n. 1-2, ene./jun. 1955.

PORTER, Michael. La ventaja competitiva de las naciones. Buenos Aires, Javier Vergara (ed.), 1991.

ROMER, Paul. Increasing returns and long run growth. Journal of Political Economy, v. 94, n. 5 , oct. 1986.

v. 98, n. 5 , oct. 1990 .

SACHS, Jeffrey D. \& LARRAIN, Felipe. Macroeconomía en la economía global. México, Prentice Hall Hispanoamericana, 1994.

SALA-I-MARTIN, Xavier. Apuntes de crecimiento económico. Barcelona, Antoni Bosch (ed.), 1994.

SOLOW, Robert M. Les évolutions de la théorie de la croissance. Paris, Problèmes Economiques, n. 2432, 19 jul. 1995.

Crecimiento y equidad. Santiago de Chile, Ed.Universitaria, 1993.

- Discurso de aceptación del Premio Nobel. En: La teoría del crecimiento. Mexico, Fondo de Cultura Económica, 1992.

A contribution to the theory of economic growth. Quarterly Journal of Economics, n. 70, feb. 1956. 
VAZQUEZ BARQUERO, Antonio. Gran empresa y desarrollo endógeno. La convergencia estratégica de las empresas y territorios ante el desafío de la competencia. Santiago de Chile, Revista EURE, n. 70, dic. 1997.

Desarrollos recientes de la política regional. La experiencia europea. Santiago de Chile, Revista EURE, n. 65, jun. 1996.

VERGARA, Rodrigo. Lucas y el crecimiento económico. Santiago de Chile. Estudios Públicos, n. 66, otoño 1997.

Nuevos modelos de crecimiento: una revisión de la literatura y algunos elementos para una estrategia de desarrollo. Santiago de Chile, Estudios Públicos, n. 43, invierno, 1991.

Carlos A. de Mattos é professor do Instituto de Estudios Urbanos, Pontificia Universidad Católica de Chile.

El autor agradece los comentarios y sugerencias recebidos de parte de Silvia Gorenstein, Luis Riffo Pérez y Verónica Silva a un texto previo sobre este tema (de Mattos, 1996), que en parte fue utilizado como base para la preparación del presente trabajo. En su conjunto, dichos comentarios y sugerencias permitieron matizar algunas afirmaciones y conclusiones e incorporar algunos aspectos entonces no considerados. 